\title{
A new class of nonlinear Gronwall-Bellman delay integral inequalities with power and its applications
}

\author{
Bo Fang ${ }^{1}$, Yujiao Liu² and Run Xu' ${ }^{1 *}$
}

"Correspondence:

xurun2005@163.com

'School of Mathematical Science,

Qufu Normal University, Qufu, China

Full list of author information is

available at the end of the article

\begin{abstract}
In this paper, we establish some new delay Gronwall-Bellman integral inequalities with power, which can be used as a convenient tool to study the qualitative properties of solutions to differential and integral equations. We also give some examples to illustrate the application of our results to obtain the estimation for the solution of the integral and differential equations.
\end{abstract}

Keywords: Delay; Integral; Inequalities; Gronwall-Bellman; Power; Nonlinear

\section{Introduction}

The field of differential equations has developed a perfect structure. Since the 20th century, inequality theory has been an active research field, a series of basic theories of inequalities have been also established [1-4]. Since for most differential equations it is difficult to find the exact form of expression, people turn to studying the qualitative nature of the solutions of differential equations, for example, the existence, uniqueness, asymptotic property, boundedness and vibration of solutions of differential equations and difference equations; inequalities have become important tools to study the qualitative properties of differential equations. In recent decades, related studies on integral inequalities have produced many results (see [5-26] and the references therein). In 1919, Gronwall [1] established the following important integral inequality for a continuous function $u$ :

$$
u(t) \leq c+\int_{a}^{t} f(\xi) u(\xi) d \xi
$$

In 1943, Bellman [2] obtained the estimation of the unknown function $u$ for some constant $c \geq 0$,

$$
u(t) \leq c \exp \left(\int_{a}^{t} f(\xi) d \xi\right)
$$

(c) The Author(s) 2021. This article is licensed under a Creative Commons Attribution 4.0 International License, which permits use, sharing, adaptation, distribution and reproduction in any medium or format, as long as you give appropriate credit to the original author(s) and the source, provide a link to the Creative Commons licence, and indicate if changes were made. The images or other third party material in this article are included in the article's Creative Commons licence, unless indicated otherwise in a credit line to the material. If material is not included in the article's Creative Commons licence and your intended use is not permitted by statutory regulation or exceeds the permitted use, you will need to obtain permission directly from the copyright holder. To view a copy of this licence, visit http://creativecommons.org/licenses/by/4.0/. 
In 1975, Pachpatte [3] studied the following integral inequality:

$$
u(t) \leq a(t)+g(t) \int_{0}^{t} f(\xi) u(\xi) d \xi
$$

where $u, f$, and $g$ are real-valued nonnegative continuous functions defined on $I=[0, \infty)$, and $a(t)$ is a positive, monotonic, nondecreasing continuous function defined on $I$.

In 1999, Owaidy et al. [5] discussed the following inequality:

$$
u(t) \leq u_{0}+\int_{0}^{t} f(\xi)\left(u^{p}(\xi)+\int_{0}^{\xi} g(\tau) u(\tau) d \tau\right) d \xi, \quad t \in[0, \infty)
$$

where $u, f$ and $g$ are real-valued nonnegative continuous functions defined on $I=[0, \infty)$.

In recent years, the time-delay dynamic equation has attracted much interest, Lipovan et al. [6] assume $u, f, g \in C\left(\left[t_{0}, T\right), R_{+}\right)$, and $\alpha \in C^{1}\left(\left[t_{0}, T\right),\left(t_{0}, T\right)\right)$ are nondecreasing with $\alpha(t) \leq t$ on $\left[t_{0}, T\right)$, and $w \in C\left(R_{+}, R_{+}\right)$are nondecreasing with $w(u)>0$ for $u>0$, then they studied the following retarded integral inequality in 2000:

$$
u(t) \leq a+\int_{t_{0}}^{t} f(\xi) w(u(\xi)) d \xi+\int_{\alpha\left(t_{0}\right)}^{\alpha(t)} g(\xi) w(u(\xi)) d \xi
$$

In 2005, Agarwal et al. [7] discussed the following $n$-term delay integral inequality:

$$
u(t) \leq a(t)+\sum_{i=1}^{n} \int_{b_{i}\left(t_{0}\right)}^{b_{i}(t)} g_{i}(t, \xi) w_{i}(t, \xi) d \xi
$$

where $u$ is a continuous and nonnegative function on $\left[t_{0}, t_{1}\right)$.

In 2011, Abdeldaim et al. [8] studied the following Gronwall-Bellman type inequality with power:

$$
u(t) \leq u_{0}+\int_{0}^{t} f(\xi) u(\xi)\left[u(\xi)+\int_{0}^{\xi} g(\lambda) u(\lambda) d \lambda\right]^{p} d \xi,
$$

where $u, f$ and $h$ are nonnegative real-valued continuous functions defined on $[0, \infty)$, and $u_{0}$ and $p$ are positive constants.

In 2019, Li et al. [9] made the following improvement on the basis of the above inequality:

$$
u(t) \leq a(t)+\int_{0}^{\sigma(t)} f(\xi) u(\xi)\left[u^{2}(\xi)+\int_{0}^{\xi} g(\lambda) u(\lambda) d \lambda\right]^{p} d \xi
$$

where $u, a, f \in C\left(R_{+}, R_{+}\right), a(t) \geq 1$, and $\alpha$ is a continuous, differentiable and increasing function on $\left[t_{0}, \infty\right)$ with $\alpha(t) \leq t, \alpha\left(t_{0}\right)=t_{0}$.

In this paper, inspired by the above work, we mainly establish the following nonlinear Gronwall-Bellman inequalities:

$$
\begin{aligned}
& u^{q}(t) \leq a(t)+\int_{t_{0}}^{\sigma(t)} f(\xi)\left[u^{\alpha}(\xi)+\int_{t_{0}}^{\xi} h(\lambda) u^{\beta}(\lambda) d \lambda\right]^{p} d \xi, \\
& u(t) \leq u_{0}+\int_{t_{0}}^{\sigma(t)} g(\xi) u^{r}(\xi)\left[u^{m}(\xi)+\int_{t_{0}}^{\xi} h(\lambda) u^{m}(\lambda) d \lambda\right]^{p} d \xi, \quad u_{0}>0,
\end{aligned}
$$




$$
\begin{aligned}
\varphi(u(t)) \leq & a(t)+\int_{t_{0}}^{t} g_{1}(t, \xi) h_{1}(u(\xi)) d \xi+\int_{t_{0}}^{t} g_{2}(t, \xi) h_{2}(u(\xi)) d \xi \\
& +\int_{t_{0}}^{t} g_{3}(t, \xi) h_{3}(u(\xi)) d \xi \\
& +\int_{t_{0}}^{t} g(t, \xi)\left(\int_{t_{0}}^{\xi} f(\xi, \theta) h_{4}(u(\theta)) d \theta\right) d \xi, \quad t_{0} \geq 0 .
\end{aligned}
$$

The structure of this paper is as follows: In Sect. 2, we illustrates some basic lemmas, which will be used in later sections. In Sect. 3, we give some new nonlinear GronwallBellman inequalities. In Sect. 4, we give two examples to illustrate the application of the obtained results in the qualitative research of differential equation solutions. In Sect. 5, we conclude our results.

\section{Preliminaries}

First, we explain some symbols will to be used: $\mathbf{R}$ denotes the set of real numbers and $\mathbf{R}_{+}=[0, \infty)$, and $C(M, S)$ denotes the class of all continuous functions on the set $M$ with range in the set $S$.

Here are some very useful lemmas.

Lemma 2.1 ([11]) Assume $a \geq 0, p \geq q \geq 0$ and $p \neq 0$, we have

$$
a^{\frac{q}{p}} \leq \frac{q}{p} K^{\frac{q-p}{p}} a+\frac{p-q}{p} K^{\frac{q}{p}}, \quad K>0
$$

We can get the following exceptional cases.

Let $K=1$, we have

$$
a^{\frac{q}{p}} \leq \frac{q}{p} a+\frac{p-q}{p}, \quad a \geq 0, p \geq q>0
$$

Let $K=1, p=1$, we have

$$
a^{q} \leq q a+(1-q), \quad a \geq 0,0<q \leq 1 .
$$

Lemma 2.2 Let $u, g, \sigma \in C\left(R_{+}, R_{+}\right), \sigma^{\prime}(t) \geq 0$ and $\sigma(t) \leq t, \sigma\left(t_{0}\right)=t_{0}, r \in(0,1], u_{0}>0$. If $u(t)$ satisfies the inequality

$$
u(t) \leq u_{0}+\int_{t_{0}}^{\sigma(t)} g(\xi) u^{r}(\xi) d \xi
$$

then

$$
u(t) \leq \exp \left(r \int_{t_{0}}^{\sigma(t)} g(\xi) d \xi\right)\left[u_{0}+\int_{t_{0}}^{\sigma(t)}(1-r) g(\xi) \exp \left(-r \int_{t_{0}}^{\xi} g(\lambda) d \lambda\right) d \xi\right]
$$

Proof We assume that

$$
v(t)=u_{0}+\int_{t_{0}}^{t} g(\xi) u^{r}(\xi) d \xi
$$


then

$$
v(\sigma(t))=u_{0}+\int_{t_{0}}^{\sigma(t)} g(\xi) u^{r}(\xi) d \xi
$$

and $u(t) \leq v(\sigma(t)) \leq v(t), v\left(\sigma\left(t_{0}\right)\right)=u_{0}$. Differentiating with respect to $t$ of (9) and using (6), we get

$$
\begin{aligned}
\sigma^{\prime}(t) v^{\prime}(\sigma(t)) & =\sigma^{\prime}(t) g(\sigma(t)) u^{r}(\sigma(t)) \\
& \leq \sigma^{\prime}(t) g(\sigma(t)) v^{r}(\sigma(t)) \\
& \leq \sigma^{\prime}(t) g(\sigma(t))[r v(\sigma(t))+(1-r)],
\end{aligned}
$$

then

$$
\sigma^{\prime}(t) v^{\prime}(\sigma(t))-r \sigma^{\prime}(t) g(\sigma(t)) v(\sigma(t)) \leq(1-r) \sigma^{\prime}(t) g(\sigma(t))
$$

Multiplying by $\exp \left(-r \int_{t_{0}}^{\sigma(t)} g(\xi) d \xi\right)$ on both sides of the above inequality, we can get

$$
\left[v(\sigma(t)) \exp \left(-r \int_{t_{0}}^{\sigma(t)} g(\xi) d \xi\right)\right]^{\prime} \leq(1-r) \sigma^{\prime}(t) g(\sigma(t)) \exp \left(-r \int_{t_{0}}^{\sigma(t)} g(\xi) d \xi\right) .
$$

Next, integrating $t$ from $t_{0}$ to $t$ for the above inequality, we get

$$
\begin{aligned}
& v(\sigma(t)) \exp \left(-r \int_{t_{0}}^{\sigma(t)} g(\xi) d \xi\right)-v\left(\sigma\left(t_{0}\right)\right) \\
& \quad \leq \int_{t_{0}}^{t}(1-r) \sigma^{\prime}(\xi) g(\sigma(\xi)) \exp \left(-r \int_{t_{0}}^{\sigma(\xi)} g(\lambda) d \lambda\right) d \xi \\
& \quad \leq \int_{t_{0}}^{\sigma(t)}(1-r) g(\xi) \exp \left(-r \int_{t_{0}}^{\xi} g(\lambda) d \lambda\right) d \xi .
\end{aligned}
$$

Since $v\left(\sigma\left(t_{0}\right)\right)=u_{0}$, we can get the estimation

$$
\begin{aligned}
u(t) & \leq v(\sigma(t)) \\
& \leq \exp \left(r \int_{t_{0}}^{\sigma(t)} g(\xi) d \xi\right)\left[u_{0}+\int_{t_{0}}^{\sigma(t)}(1-r) g(\xi) \exp \left(-r \int_{t_{0}}^{\xi} g(\lambda) d \lambda\right) d \xi\right] .
\end{aligned}
$$

This completes the proof.

\section{Main result and proof}

In this section, we establish and prove a new class of nonlinear Gronwall-Bellman type delay integral inequalities with power.

Theorem 3.1 We assume $u, a, f, h \in C\left(R_{+}, R_{+}\right)$, and let $\sigma(t) \in C\left[t_{0}, \infty\right), \sigma^{\prime}(t) \geq 0$ and $\sigma(t) \leq t, \sigma\left(t_{0}\right)=t_{0} . q \geq \alpha>0, q \geq \beta>0, p \in(0,1]$ and $q \geq p$. If $u$ satisfies the inequal- 
ity (1), then we can get

$$
\begin{aligned}
u(t) \leq & {\left[a(t)+B(t) \exp \left(\int_{t_{0}}^{\sigma(t)} \frac{p \alpha}{q} f(\xi) d \xi\right.\right.} \\
& \left.\left.+\int_{t_{0}}^{\sigma(t)} \frac{p \beta}{q} f(\xi)\left(\int_{t_{0}}^{\xi} h(\lambda) d \lambda\right) d \xi\right)\right]^{\frac{1}{q}}, \quad \forall t \in R_{+},
\end{aligned}
$$

where

$$
\begin{aligned}
B(t)= & \int_{t_{0}}^{\sigma(t)} f(\xi)\left((1-p)+\frac{p \alpha}{q} a(\xi)+\frac{q p-p \alpha}{q}\right) d \xi \\
& +\int_{t_{0}}^{\sigma(t)} f(\xi)\left[\int_{t_{0}}^{\xi} h(\lambda)\left(\frac{p \alpha}{q} a(\lambda)+\frac{q p-p \beta}{q}\right) d \lambda\right] d \xi
\end{aligned}
$$

Proof Using (6), we have

$$
\left[u^{\alpha}(s)+\int_{t_{0}}^{s} h(\lambda) u^{\beta}(\lambda) d \lambda\right]^{p} \leq p\left[u^{\alpha}(s)+\int_{t_{0}}^{s} h(\lambda) u^{\beta}(\lambda) d \lambda\right]+(1-p) .
$$

Plugging (11) into (1), we can get

$$
u^{q}(t) \leq a(t)+\int_{t_{0}}^{\sigma(t)} f(\xi)\left[p\left(u^{\alpha}(\xi)+\int_{t_{0}}^{\xi} h(\lambda) u^{\beta}(\lambda) d \lambda\right)+(1-p)\right] d \xi
$$

Now, we define $v(t)$ by

$$
v(t)=\int_{t_{0}}^{\sigma(t)} f(\xi)\left[p\left(u^{\alpha}(\xi)+\int_{t_{0}}^{\xi} h(\lambda) u^{\beta}(\lambda) d \lambda\right)+(1-p)\right] d \xi
$$

then $v(t)$ is a nondecreasing function, using (12) and (13), we obtain

$$
u(t) \leq[a(t)+v(t)]^{\frac{1}{q}} .
$$

Using (5), from the above inequality we get

$$
u^{\alpha}(t) \leq(a(t)+v(t))^{\frac{\alpha}{q}} \leq \frac{\alpha}{q}(a(t)+v(t))+\left(1-\frac{\alpha}{q}\right)
$$

and

$$
u^{\beta}(t) \leq(a(t)+v(t))^{\frac{\beta}{q}} \leq \frac{\beta}{q}(a(t)+v(t))+\left(1-\frac{\beta}{q}\right) .
$$

Plugging (14) and (15) into (13), we can obtain

$$
\begin{aligned}
v(t) \leq & \int_{t_{0}}^{\sigma(t)} f(\xi)\left[p \left(\frac{\alpha}{q}(a(\xi)+v(\xi))+\left(1-\frac{\alpha}{q}\right)\right.\right. \\
& \left.\left.+\int_{t_{0}}^{\xi} h(\lambda)\left(\frac{\beta}{q}(a(\lambda)+v(\lambda))+\left(1-\frac{\beta}{q}\right)\right) d \lambda\right)+(1-p)\right] d \xi
\end{aligned}
$$




$$
\begin{aligned}
\leq & \int_{t_{0}}^{\sigma(t)} f(\xi)\left((1-p)+\frac{p \alpha}{q} a(\xi)+\frac{q p-p \alpha}{q}\right) d \xi \\
& +\int_{t_{0}}^{\sigma(t)} f(\xi)\left[\int_{t_{0}}^{\xi} h(\lambda)\left(\frac{p \alpha}{q} a(\lambda)+\frac{q p-p \beta}{q}\right) d \lambda\right] d \xi \\
& +\int_{t_{0}}^{\sigma(t)} \frac{p \alpha}{q} f(\xi) v(\xi) d \xi+\int_{t_{0}}^{\sigma(t)} \frac{p \beta}{q} f(\xi)\left(\int_{t_{0}}^{\xi} h(\lambda) v(\lambda) d \lambda\right) d \xi \\
\leq & B(t)+\int_{t_{0}}^{\sigma(t)} \frac{p \alpha}{q} f(\xi) v(\xi) d \xi+\int_{t_{0}}^{\sigma(t)} \frac{p \beta}{q} f(\xi)\left(\int_{t_{0}}^{\xi} h(\lambda) v(\lambda) d \lambda\right) d \xi \\
\leq & B(T)+\int_{t_{0}}^{\sigma(t)} \frac{p \alpha}{q} f(\xi) v(\xi) d \xi+\int_{t_{0}}^{\sigma(t)} \frac{p \beta}{q} f(\xi)\left(\int_{t_{0}}^{\xi} h(\lambda) v(\lambda) d \lambda\right) d \xi
\end{aligned}
$$

where $t \in\left[t_{0}, T\right], T \in R_{+}$, and

$$
\begin{aligned}
B(t)= & \int_{t_{0}}^{\sigma(t)} f(\xi)\left((1-p)+\frac{p \alpha}{q} a(\xi)+\frac{q p-p \alpha}{q}\right) d \xi \\
& +\int_{t_{0}}^{\sigma(t)} f(\xi)\left[\int_{t_{0}}^{\xi} h(\lambda)\left(\frac{p \alpha}{q} a(\lambda)+\frac{q p-p \beta}{q}\right) d \lambda\right] d \xi
\end{aligned}
$$

Let

$$
y(t)=B(T)+\int_{t_{0}}^{\sigma(t)} \frac{p \alpha}{q} f(\xi) v(\xi) d \xi+\int_{t_{0}}^{\sigma(t)} \frac{p \beta}{q} f(\xi)\left(\int_{t_{0}}^{\xi} h(\lambda) v(\lambda) d \lambda\right) d \xi .
$$

Then we can get $y(t)$ is a nondecreasing and positive function, and $v(t) \leq y(t), y\left(t_{0}\right)=B(T)$.

Differentiating $y(t)$ with respect to $t$ and using $\sigma(t) \leq t$, we have

$$
\begin{aligned}
y^{\prime}(t) & =\frac{p \alpha}{q} \sigma^{\prime}(t) f(\sigma(t)) v(\sigma(t))+\frac{p \beta}{q} \sigma^{\prime}(t) f(\sigma(t)) \int_{t_{0}}^{\sigma(t)} h(\xi) v(\xi) d \xi \\
& \leq v(t)\left(\frac{p \alpha}{q} \sigma^{\prime}(t) f(\sigma(t))+\frac{p \beta}{q} \sigma^{\prime}(t) f(\sigma(t)) \int_{t_{0}}^{\sigma(t)} h(\xi) d \xi\right) \\
& \leq y(t)\left(\frac{p \alpha}{q} \sigma^{\prime}(t) f(\sigma(t))+\frac{p \beta}{q} \sigma^{\prime}(t) f(\sigma(t)) \int_{t_{0}}^{\sigma(t)} h(\xi) d \xi\right) .
\end{aligned}
$$

From the above inequality we get

$$
\frac{y^{\prime}(t)}{y(t)} \leq \frac{p \alpha}{q} \sigma^{\prime}(t) f(\sigma(t))+\frac{p \beta}{q} \sigma^{\prime}(t) f(\sigma(t)) \int_{t_{0}}^{\sigma(t)} h(\xi) d \xi .
$$

Integrating both side of the above inequality from $t_{0}$ to $t$, then we can obtain the estimation for $y(t)$ :

$$
y(t) \leq B(T) \exp \left(\int_{t_{0}}^{\sigma(t)} \frac{p \alpha}{q} f(s) d s+\int_{t_{0}}^{\sigma(t)} \frac{p \beta}{q} f(s)\left(\int_{t_{0}}^{s} h(\lambda) d \lambda\right) d s\right),
$$

by $v(t) \leq y(t)$ and $u(t) \leq[a(t)+v(t)]^{\frac{1}{q}}$, we can obtain

$$
u(t) \leq\left[a(t)+B(T) \exp \left(\int_{t_{0}}^{\sigma(t)} \frac{p \alpha}{q} f(s) d s+\int_{t_{0}}^{\sigma(t)} \frac{p \beta}{q} f(s)\left(\int_{t_{0}}^{s} h(\lambda) d \lambda\right) d s\right)\right]^{\frac{1}{q}} .
$$


Thus

$$
u(T) \leq\left[a(T)+B(T) \exp \left(\int_{t_{0}}^{\sigma(t)} \frac{p \alpha}{q} f(s) d s+\int_{t_{0}}^{\sigma(t)} \frac{p \beta}{q} f(s)\left(\int_{t_{0}}^{s} h(\lambda) d \lambda\right) d s\right)\right]^{\frac{1}{q}} .
$$

Because of the arbitrariness of $T$, we can obtain

$$
u(t) \leq\left[a(t)+B(t) \exp \left(\int_{t_{0}}^{\sigma(t)} \frac{p \alpha}{q} f(s) d s+\int_{t_{0}}^{\sigma(t)} \frac{p \beta}{q} f(s)\left(\int_{t_{0}}^{s} h(\lambda) d \lambda\right) d s\right)\right]^{\frac{1}{q}} .
$$

The proof is complete.

Remark 1 If $q=1$, Theorem 3.1 reduces to Theorem 2.1 in [9]. If $q=p, a(t)=x_{0}, \sigma(t)=t$, $p=\beta=1, \alpha=q$, Theorem 3.1 reduces to Theorem 2.3 in [10]. If $q=1, a(t)=x_{0}, \sigma(t)=t$, $p=\beta=1, \alpha=2-p, \beta=q$, Theorem 3.1 reduces to Theorem 2.5 in [10]. If $q=1, a(t)=x_{0}$, $\sigma(t)=t, \alpha=p, \beta=2 p-1$, Theorem 3.1 reduces to Theorem 2.8 in [10].

Theorem 3.2 We assume $u, g, h \in C\left(R_{+}, R_{+}\right), \sigma(t) \in\left[t_{0}, \infty\right), \sigma^{\prime}(t) \geq 0, \sigma(t) \leq t, \sigma\left(t_{0}\right)=t_{0}$, $r \in(0,1], p>1, m>1$. If $u$ satisfies the inequality (2), then

$$
\begin{aligned}
u(t) \leq & \exp \left(r \int_{t_{0}}^{\sigma(t)} g(\xi) \beta(\xi) d \xi\right) \\
& \times\left[u_{0}+\int_{t_{0}}^{\sigma(t)}(1-r) g(\xi) \beta(\xi) \exp \left(-r \int_{t_{0}}^{\xi} g(\lambda) \beta(\lambda) d \lambda\right) d \xi\right],
\end{aligned}
$$

where

$$
\begin{aligned}
& \beta(t)=\left[\frac{\Omega(t)}{1+(1-r-m p) \int_{t_{0}}^{t} g(\xi) \Omega(\xi) d \xi}\right]^{\frac{m p}{m p+r-1}}, \\
& \Omega(t)=u_{0}^{r+m p-1} \exp \left(\frac{r+m p-1}{m} \int_{t_{0}}^{t} h(\xi) d \xi\right), \\
& 1+(1-r-m p) \int_{t_{0}}^{\sigma(t)} g(\xi) \Omega(\xi) d \xi>0 .
\end{aligned}
$$

Proof First, we denote

$$
J(t)=u_{0}+\int_{t_{0}}^{t} g(\xi) u^{r}(\xi)\left[u^{m}(\xi)+\int_{t_{0}}^{\xi} h(\lambda) u^{m}(\lambda) d \lambda\right]^{p} d \xi
$$

and $J(t)$ is a nondecreasing and nonnegative continuous function, then

$$
J(\sigma(t))=u_{0}+\int_{t_{0}}^{\sigma(t)} g(\xi) u^{r}(\xi)\left[u^{m}(\xi)+\int_{t_{0}}^{\xi} h(\lambda) u^{m}(\lambda) d \lambda\right]^{p} d \xi
$$


and $u(t) \leq J(\sigma(t)) \leq J(t), J\left(\sigma\left(t_{0}\right)\right)=J\left(t_{0}\right)=u_{0}$. Differentiating with respect to $t$ of the above equation, we get

$$
\begin{aligned}
\sigma^{\prime}(t) \frac{d J(\sigma(t))}{d \sigma} & =\sigma^{\prime}(t) g(\sigma(t)) u^{r}(\sigma(t))\left[u^{m}(\sigma(t))+\int_{t_{0}}^{\sigma(t)} h(\lambda) u^{m}(\lambda) d \lambda\right]^{p} \\
& \leq \sigma^{\prime}(t) g(\sigma(t)) J^{r}(\sigma(t))\left[J^{m}(\sigma(t))+\int_{t_{0}}^{\sigma(t)} h(\lambda) J^{m}(\lambda) d \lambda\right]^{p} \\
& =\sigma^{\prime}(t) g(\sigma(t)) J^{r}(\sigma(t)) Y^{p}(\sigma(t)),
\end{aligned}
$$

which means

$$
\frac{d J(\sigma(t))}{d \sigma} \leq g(\sigma(t)) J^{r}(\sigma(t)) Y^{p}(\sigma(t))
$$

where $Y(t)=J^{m}(t)+\int_{t_{0}}^{t} h(\lambda) J^{m}(\lambda) d \lambda$, then $Y(\sigma(t))=J^{m}(\sigma(t))+\int_{t_{0}}^{\sigma(t)} h(\lambda) J^{m}(\lambda) d \lambda$, hence $Y\left(\sigma\left(t_{0}\right)\right)=J^{m}\left(\sigma\left(t_{0}\right)\right)=J^{m}\left(t_{0}\right)=u_{0}{ }^{m}$, we can conclude that

$$
J(\sigma(t)) \leq Y^{\frac{1}{m}}(\sigma(t))
$$

Differentiating with respect to $t$ of $Y(\sigma(t))$, we get

$$
\begin{aligned}
\sigma^{\prime}(t) \frac{d Y(\sigma(t))}{d \sigma} & =m \sigma^{\prime}(t) J^{m-1}(\sigma(t)) \frac{d J(\sigma(t))}{d \sigma}+\sigma^{\prime}(t) h(\sigma(t)) J^{m}(\sigma(t)) \\
& \leq m \sigma^{\prime}(t) J^{m-1}(\sigma(t))\left[g(\sigma(t)) J^{r}(\sigma(t)) Y^{p}(\sigma(t))\right]+\sigma^{\prime}(t) h(\sigma(t)) J^{m}(\sigma(t)) \\
& \leq m \sigma^{\prime}(t) Y^{\frac{m-1}{m}}(\sigma(t))\left[g(\sigma(t)) Y^{\frac{r+m p}{m}}(\sigma(t))\right]+\sigma^{\prime}(t) h(\sigma(t)) Y(\sigma(t)),
\end{aligned}
$$

then

$$
\frac{d Y(\sigma(t))}{d \sigma} \leq m g(\sigma(t)) Y^{\frac{r+m p+m-1}{m}}(\sigma(t))+h(\sigma(t)) Y(\sigma(t))
$$

from the above inequality, we can get

$$
Y^{\frac{1-r-m p-m}{m}}(\sigma(t)) \frac{d Y(\sigma(t))}{d \sigma}-h(\sigma(t)) Y^{\frac{1-r-m p}{m}}(\sigma(t)) \leq m g(\sigma(t)) .
$$

Denote

$$
\Gamma(t)=Y^{\frac{1-r-m p}{m}}(t)
$$

then $\Gamma(\sigma(t))=Y^{\frac{1-r-m p}{m}}(\sigma(t)), \frac{d \Gamma(\sigma(t))}{d t}=\frac{1-r-m p}{m} Y^{\frac{1-m-r-m p}{m}}(\sigma(t)) \sigma^{\prime}(t) \frac{d Y(\sigma(t))}{d \sigma}$, and $\Gamma\left(\sigma\left(t_{0}\right)\right)=$ $Y^{\frac{1-r-m p}{m}}\left(\sigma\left(t_{0}\right)\right)=u_{0}^{1-r-m p}$, from $1-r-m p<0$ and (19), we have

$$
\frac{d \Gamma(\sigma(t))}{d t}-\frac{1-r-m p}{m} \sigma^{\prime}(t) \Gamma(\sigma(t)) h(\sigma(t)) \geq(1-r-m p) \sigma^{\prime}(t) g(\sigma(t)),
$$


Multiplying by $\exp \left(\frac{r+m p-1}{m} \int_{t_{0}}^{\sigma(t)} h(\xi) d \xi\right)$ on both sides of the above inequality, we get

$$
\begin{aligned}
& {\left[\Gamma(\sigma(t)) \exp \left(\frac{r+m p-1}{m} \int_{t_{0}}^{\sigma(t)} h(\xi) d \xi\right)\right]^{\prime}} \\
& \quad \geq(1-r-m p) \sigma^{\prime}(t) g(\sigma(t)) \exp \left(\frac{r+m p-1}{m} \int_{t_{0}}^{\sigma(t)} h(\xi) d \xi\right) .
\end{aligned}
$$

Integrating both sides of the above inequality from $t_{0}$ to $t$, we can get

$$
\begin{aligned}
& \Gamma(\sigma(t)) \exp \left(\frac{r+m p-1}{m} \int_{t_{0}}^{\sigma(t)} h(\xi) d \xi\right)-\Gamma\left(\sigma\left(t_{0}\right)\right) \\
& \quad \geq(1-r-m p) \int_{t_{0}}^{t} \sigma^{\prime}(\xi) g(\sigma(\xi)) \exp \left(\frac{r+m p-1}{m} \int_{t_{0}}^{\sigma(\xi)} h(\lambda) d \lambda\right) d \xi \\
& \quad=(1-r-m p) \int_{t_{0}}^{\sigma(t)} g(\xi) \exp \left(\frac{r+m p-1}{m} \int_{t_{0}}^{\xi} h(\lambda) d \lambda\right) d \xi .
\end{aligned}
$$

Since $\Gamma\left(\sigma\left(t_{0}\right)\right)=Y^{\frac{1-r-m p}{m}}\left(\sigma\left(t_{0}\right)\right)=u_{0}^{1-r-m p}$, we get

$$
\Gamma(\sigma(t)) \geq \frac{u_{0}^{1-r-m p}+(1-r-m p) \int_{t_{0}}^{\sigma(t)} g(\xi) \exp \left(\frac{r+m p-1}{m} \int_{t_{0}}^{\xi} h(\lambda) d \lambda\right) d \xi}{\exp \left(\frac{r+m p-1}{m} \int_{t_{0}}^{\sigma(t)} h(\xi) d \xi\right)}
$$

which means

$$
\Gamma(\sigma(t)) \geq \frac{1+(1-r-m p) u_{0}^{r+m p-1} \int_{t_{0}}^{\sigma(t)} g(\xi) \exp \left(\frac{r+m p-1}{m} \int_{t_{0}}^{\xi} h(\lambda) d \lambda\right) d \xi}{u_{0}^{r+m p-1} \exp \left(\frac{r+m p-1}{m} \int_{t_{0}}^{\sigma(t)} h(\xi) d \xi\right)}
$$

Let $\Omega(t)=u_{0}^{r+m p-1} \exp \left(\frac{r+m p-1}{m} \int_{t_{0}}^{t} h(\xi) d \xi\right)$, using $\Gamma(\sigma(t))=Y^{\frac{1-r-m p}{m}}(\sigma(t))$, we can get

$$
Y^{p}(\sigma(t)) \leq\left[\frac{\Omega(\sigma(t))}{1+(1-r-m p) \int_{t_{0}}^{\sigma(t)} g(\xi) \Omega(\xi) d \xi}\right]^{\frac{m p}{m p+r-1}}
$$

where $1+(1-r-m p) \int_{t_{0}}^{\sigma(t)} g(\xi) \Omega(\xi) d \xi>0$.

By the definition of $\beta(t)$, plugging the above inequality into (18), we can get

$$
\frac{d J(\sigma(t))}{d \sigma} \leq g(\sigma(t)) \beta(\sigma(t)) J^{r}(\sigma(t))
$$

Integrating both sides of the above inequality from $t$ to $t_{0}$, we get

$$
J(\sigma(t)) \leq u_{0}+\int_{t_{0}}^{\sigma(t)} g(\xi) \beta(\xi) J^{r}(\xi) d \xi
$$


Therefore, from Lemma 2.2 we can get

$$
\begin{aligned}
u(t) \leq & J(\sigma(t)) \\
\leq & \exp \left(r \int_{t_{0}}^{\sigma(t)} g(\xi) \beta(\xi) d \xi\right) \\
& \times\left[u_{0}+\int_{t_{0}}^{\sigma(t)}(1-r) g(\xi) \beta(\xi) \exp \left(-r \int_{t_{0}}^{\xi} g(\lambda) \beta(\lambda) d \lambda\right) d \xi\right] .
\end{aligned}
$$

The proof is completed.

Remark 2 If $u_{0}=x_{0}, \sigma(t)=t$, and $r=m=1$, Theorem 3.2 reduces to Theorem 3.2 in [8].

In the following, we discuss the inequality (3). First we assume that the following conditions are satisfied;

$\left(C_{1}\right) \varphi(u)$ is a positive continuous and strictly increasing function on $[0, \infty)$.

$\left(C_{2}\right) h_{j}(u),(j=1,2,3,4)$ are positive, continuous and increasing functions on $[0, \infty)$, and $\frac{h_{j+1}(t)}{h_{j}(t)},(j=1,2,3)$ are nondecreasing functions. Moreover, let

$$
y_{j}(t)=\frac{h_{j}(t)}{h_{1}(t)}, \quad j=1,2,3,4,
$$

thus $y_{j}(t)$ are nondecreasing functions, $y_{1}(t)=1$ and

$$
\frac{y_{j+1}(t)}{y_{j}(t)}=\frac{h_{j+1}(t)}{h_{j}(t)}, \quad j=1,2,3,
$$

then $\frac{y_{j+1}(t)}{y_{j}(t)}$ are nondecreasing, positive and continuous functions.

$\left(C_{3}\right)$ We define the following functions:

$$
H_{j}(u)=\int_{1}^{u} \frac{d \xi}{h_{j}\left(\varphi^{-1}(\xi)\right)}, \quad j=1,2,3,4
$$

Then $H_{j}$ are positive continuous and strictly increasing functions on $[0, \infty)$. We assume that $H_{j}^{-1}$ define the inverse function of $H_{j}$, which are also continuous nondecreasing functions.

$\left(C_{4}\right) a(t)$ is a continuous function on $\left[t_{0}, \infty\right), a(t) \geq 0, a\left(t_{0}\right) \neq 0$, and $g_{j}(t, \xi)(j=1,2,3)$ and $f(t, \xi)$ are continuous functions on $\left[t_{0}, \infty\right) \times\left[t_{0}, \infty\right)$.

$\left(C_{5}\right)$ We assume that $g(t, \xi), f(t, \xi)$ are nondecreasing and continuous functions on $\left[t_{0}, \infty\right) \times\left[t_{0}, \infty\right)$, and

$$
g_{4}(t, \xi)=\int_{\xi}^{t} g(t, \theta) f(\theta, \xi) d \theta, \quad t, \xi \in\left[t_{0}, \infty\right)
$$

$\left(C_{6}\right) a(t)+\sum_{j=1}^{4} g_{j}(t, \xi) h_{j}(u(\xi))>0$.

Theorem 3.3 Suppose the conditions $\left(C_{1}\right)-\left(C_{6}\right)$ are satisfied, $u$ is a positive and continuous function on $t \geq t_{0} \geq 0$, if $u$ satisfies (3), we can get the following estimation for $u$ :

$$
u(t) \leq \varphi^{-1}\left(H_{4}^{-1}\left(A_{5}(t)\right)\right), \quad t \in\left[t_{0}, \infty\right)
$$


where

$$
\begin{aligned}
& A_{1}(t)=a(t), \\
& A_{2}(t)=H_{1}(a(t))+\int_{t_{0}}^{t} g_{1}(t, \xi) d \xi, \\
& A_{j+1}(t)=H_{j}\left(H_{j-1}^{-1}\left(A_{j}(t)\right)\right)+\int_{t_{0}}^{t} g_{j}(t, \xi) d \xi, \quad j=2,3,4 .
\end{aligned}
$$

Proof Since $g(t, \xi), f(t, \xi), h_{4}(u(t))$ are nondecreasing and continuous functions, by $\left(C_{5}\right)$, we can get

$$
\begin{aligned}
& \int_{t_{0}}^{t} g(t, \xi)\left(\int_{t_{0}}^{\xi} f(\xi, \theta) h_{4}(u(\theta)) d \theta\right) d \xi \\
& =\int_{t_{0}}^{t} h_{4}(u(\theta)) \int_{\theta}^{t} g(t, \xi) f(\xi, \theta) d \xi d \theta \\
& =\int_{t_{0}}^{t} h_{4}(u(\xi)) \int_{\xi}^{t} g(t, \theta) f(\theta, \xi) d \theta d \xi \\
& =\int_{t_{0}}^{t} g_{4}(t, \xi) h_{4}(u(\xi)) d \xi,
\end{aligned}
$$

where the first equality is obtained by swapping the order of double integral, the second equality is obtained by $\theta=\xi$, the third equality is a simplification of the above equation obtained by $\left(C_{5}\right)$. Plugging (24) into (3), we can write (3) as

$$
\varphi(u(t)) \leq a(t)+\sum_{j=1}^{4} \int_{t_{0}}^{t} g_{j}(t, \xi) h_{j}(u(\xi)) d \xi .
$$

For any fixed $T \in\left[t_{0}, \infty\right)$ and for $t \in\left[t_{0}, T\right]$, from the above inequality, we have

$$
\varphi(u(t)) \leq a(t)+\sum_{j=1}^{4} \int_{t_{0}}^{t} g_{j}(T, \xi) h_{j}(u(\xi)) d \xi
$$

We assume that

$$
z_{1}(t)=a(t)+\sum_{j=1}^{4} \int_{t_{0}}^{t} g_{j}(T, \xi) h_{j}(u(\xi)) d \xi
$$

thus $z_{1}(t)$ is a nondecreasing and nonnegative continuous function, and we have $\varphi(u(t)) \leq$ $z_{1}(t), u(t) \leq \varphi^{-1}\left(z_{1}(t)\right), z_{1}\left(t_{0}\right)=a\left(t_{0}\right), z_{1}(t) \geq a(t)$. We can take the derivative with respect to $t$ in (25), then

$$
z_{1}^{\prime}(t)=a^{\prime}(t)+\sum_{j=1}^{4} g_{j}(T, t) h_{j}(u(t)) .
$$


Multiplying both sides of the above inequality by $\frac{1}{h_{1}\left(\varphi^{-1}\left(z_{1}(t)\right)\right)}$, meanwhile using (20), we have

$$
\begin{aligned}
\frac{z_{1}^{\prime}(t)}{h_{1}\left(\varphi^{-1}\left(z_{1}(t)\right)\right)} & =\frac{a^{\prime}(t)+\sum_{j=1}^{4} h_{j}(u(t)) g_{j}(T, t)}{h_{1}\left(\varphi^{-1}\left(z_{1}(t)\right)\right)} \\
& \leq \frac{a^{\prime}(t)+\sum_{j=1}^{4} h_{j}\left(\varphi^{-1}\left(z_{1}(t)\right)\right) g_{j}(T, t)}{h_{1}\left(\varphi^{-1}\left(z_{1}(t)\right)\right)} \\
& =\frac{a^{\prime}(t)}{h_{1}\left(\varphi^{-1}\left(z_{1}(t)\right)\right)}+g_{1}(T, t)+\frac{\sum_{j=2}^{4} h_{j}\left(\varphi^{-1}\left(z_{1}(t)\right)\right) g_{j}(T, t)}{h_{1}\left(\varphi^{-1}\left(z_{1}(t)\right)\right)} \\
& \leq \frac{a^{\prime}(t)}{h_{1}\left(\varphi^{-1}(a(t))\right)}+g_{1}(T, t)+\sum_{j=1}^{3} g_{j+1}(T, t) y_{j+1}\left(\varphi^{-1}\left(z_{1}(t)\right)\right),
\end{aligned}
$$

integrating both sides of the above inequality from $t_{0}$ to $t$, and using the definition of (22), we obtain

$$
\begin{aligned}
H_{1}\left(z_{1}(t)\right)-H_{1}\left(z_{1}\left(t_{0}\right)\right) \leq & H_{1}(a(t))-H_{1}\left(a\left(t_{0}\right)\right)+\int_{t_{0}}^{t} g_{1}(T, \xi) d \xi \\
& +\sum_{j=1}^{3} \int_{t_{0}}^{t} g_{j+1}(T, \xi) y_{j+1}\left(\varphi^{-1}\left(z_{1}(\xi)\right)\right) d \xi,
\end{aligned}
$$

which means

$$
H_{1}\left(z_{1}(t)\right) \leq H_{1}(a(t))+\int_{t_{0}}^{t} g_{1}(T, \xi) d \xi+\sum_{j=1}^{3} \int_{t_{0}}^{t} g_{j+1}(T, \xi) y_{j+1}\left(\varphi^{-1}\left(z_{1}(\xi)\right)\right) d \xi
$$

We assume that

$$
\eta_{1}(t)=H_{1}\left(z_{1}(t)\right)
$$

and

$$
A_{2}(t)=H_{1}\left(A_{1}(t)\right)+\int_{t_{0}}^{t} g_{1}(T, \xi) d \xi
$$

from (27) and (28), (26) can be written as

$$
\begin{aligned}
\eta_{1}(t) & \leq A_{2}(t)+\sum_{j=1}^{3} \int_{t_{0}}^{t} g_{j+1}(T, \xi) y_{j+1}\left(\varphi^{-1}\left(z_{1}(\xi)\right)\right) d \xi \\
& =A_{2}(t)+\sum_{j=1}^{3} \int_{t_{0}}^{t} g_{j+1}(T, \xi) y_{j+1}\left(\varphi^{-1}\left(H_{1}^{-1}\left(\eta_{1}(\xi)\right)\right)\right) d \xi .
\end{aligned}
$$

Then we assume that

$$
z_{2}(t)=A_{2}(t)+\sum_{j=1}^{3} \int_{t_{0}}^{t} g_{j+1}(T, \xi) y_{j+1}\left(\varphi^{-1}\left(H_{1}^{-1}\left(\eta_{1}(\xi)\right)\right)\right) d \xi
$$


thus $z_{2}(t)$ is a nondecreasing and continuous function, and $\eta_{1}(t) \leq z_{2}(t), z_{2}\left(t_{0}\right)=A_{2}\left(t_{0}\right)$, $A_{2}(t) \leq z_{2}(t)$.

We define a function as

$$
Y_{j+1}(u)=\int_{0}^{u} \frac{y_{j}\left(\varphi^{-1}\left(H_{j}^{-1}(\xi)\right)\right)}{y_{j+1}\left(\varphi^{-1}\left(H_{j}^{-1}(\xi)\right)\right)} d \xi, \quad j=1,2,3
$$

then, by (21) and (30), we can obtain

$$
\begin{aligned}
Y_{j+1}(u) & =\int_{0}^{u} \frac{y_{j}\left(\varphi^{-1}\left(H_{j}^{-1}(\xi)\right)\right)}{y_{j+1}\left(\varphi^{-1}\left(H_{j}^{-1}(\xi)\right)\right)} d \xi \\
& =\int_{0}^{u} \frac{h_{j}\left(\varphi^{-1}\left(H_{j}^{-1}(\xi)\right)\right)}{h_{j+1}\left(\varphi^{-1}\left(H_{j}^{-1}(\xi)\right)\right)} d \xi \\
& =\int_{H_{j}^{-1}(0)}^{H_{j}^{-1}(u)} \frac{h_{j}\left(\varphi^{-1}(t)\right)}{h_{j+1}\left(\varphi^{-1}(t)\right)}\left(H_{j}(t)\right)^{\prime} d t \\
& =\int_{1}^{H_{j}^{-1}(u)} \frac{1}{h_{j+1}\left(\varphi^{-1}(t)\right)} d t \\
& =H_{j+1}\left(H_{j}^{-1}(u)\right), \quad j=1,2,3,
\end{aligned}
$$

from (22), we have $H_{j}(1)=0, H_{j}^{-1}(0)=1,\left(H_{j}(t)\right)^{\prime}=\frac{1}{h_{j}\left(\varphi^{-1}(t)\right)}$. Taking the derivative with respect to $t$ in (29), then multiplying both sides of it by $\frac{1}{y_{2}\left(\varphi^{-1}\left(H_{1}^{-1}\left(z_{2}(t)\right)\right)\right)}$, by $y_{1}(t)=1$, we have

$$
\begin{aligned}
\frac{z_{2}{ }^{\prime}(t)}{y_{2}\left(\varphi^{-1}\left(H_{1}^{-1}\left(z_{2}(t)\right)\right)\right)}= & \frac{A_{2}{ }^{\prime}(t)+\sum_{j=1}^{3} g_{j+1}(T, t) y_{j+1}\left(\varphi^{-1}\left(H_{1}^{-1}\left(\eta_{1}(t)\right)\right)\right)}{y_{2}\left(\varphi^{-1}\left(H_{1}^{-1}\left(z_{2}(t)\right)\right)\right)} \\
\leq & \frac{A_{2}{ }^{\prime}(t)+\sum_{j=1}^{3} g_{j+1}(T, t) y_{j+1}\left(\varphi^{-1}\left(H_{1}^{-1}\left(z_{2}(t)\right)\right)\right)}{y_{2}\left(\varphi^{-1}\left(H_{1}^{-1}\left(z_{2}(t)\right)\right)\right)} \\
= & \frac{A_{2}{ }^{\prime}(t)}{y_{2}\left(\varphi^{-1}\left(H_{1}^{-1}\left(z_{2}(t)\right)\right)\right)}+g_{2}(T, t) \\
& +\sum_{j=2}^{3} \frac{g_{j+1}(T, t) y_{j+1}\left(\varphi^{-1}\left(H_{1}^{-1}\left(z_{2}(t)\right)\right)\right)}{y_{2}\left(\varphi^{-1}\left(H_{1}^{-1}\left(z_{2}(t)\right)\right)\right)} .
\end{aligned}
$$

Again, integrating both sides of (31) from $t_{0}$ to $t$, and by the definition in (30) and (20), we can obtain

$$
\begin{aligned}
Y_{2}\left(z_{2}(t)\right)-Y_{2}\left(z_{2}\left(t_{0}\right)\right) \leq & \int_{t_{0}}^{t} \frac{A_{2}{ }^{\prime}(\xi)}{y_{2}\left(\varphi^{-1}\left(H_{1}^{-1}\left(z_{2}(\xi)\right)\right)\right)} d \xi+\int_{t_{0}}^{t} g_{2}(T, \xi) d \xi \\
& +\sum_{j=2}^{3} \int_{t_{0}}^{t} \frac{g_{j+1}(T, \xi) y_{j+1}\left(\varphi^{-1}\left(H_{1}^{-1}\left(z_{2}(\xi)\right)\right)\right)}{y_{2}\left(\varphi^{-1}\left(H_{1}^{-1}\left(z_{2}(\xi)\right)\right)\right)} d \xi \\
\leq & \int_{t_{0}}^{t} \frac{A_{2}{ }^{\prime}(\xi)}{y_{2}\left(\varphi^{-1}\left(H_{1}^{-1}\left(A_{2}(\xi)\right)\right)\right)} d \xi+\int_{t_{0}}^{t} g_{2}(T, \xi) d \xi \\
& +\sum_{j=2}^{3} \int_{t_{0}}^{t} \frac{g_{j+1}(T, \xi) y_{j+1}\left(\varphi^{-1}\left(H_{1}^{-1}\left(z_{2}(\xi)\right)\right)\right)}{y_{2}\left(\varphi^{-1}\left(H_{1}^{-1}\left(z_{2}(\xi)\right)\right)\right)} d \xi
\end{aligned}
$$




$$
\begin{aligned}
\leq & Y_{2}\left(A_{2}(t)\right)-Y_{2}\left(A_{2}\left(t_{0}\right)\right)+\int_{t_{0}}^{t} g_{2}(T, \xi) d \xi \\
& +\sum_{j=2}^{3} \int_{t_{0}}^{t} \frac{g_{j+1}(T, \xi) y_{j+1}\left(\varphi^{-1}\left(H_{1}^{-1}\left(z_{2}(\xi)\right)\right)\right)}{y_{2}\left(\varphi^{-1}\left(H_{1}^{-1}\left(z_{2}(\xi)\right)\right)\right)} d \xi,
\end{aligned}
$$

using $z_{2}\left(t_{0}\right)=A_{2}\left(t_{0}\right)$, we have

$$
Y_{2}\left(z_{2}(t)\right) \leq Y_{2}\left(A_{2}(t)\right)+\int_{t_{0}}^{t} g_{2}(T, \xi) d \xi+\sum_{j=2}^{3} \int_{t_{0}}^{t} \frac{g_{j+1}(T, \xi) y_{j+1}\left(\varphi^{-1}\left(H_{1}^{-1}\left(z_{2}(\xi)\right)\right)\right)}{y_{2}\left(\varphi^{-1}\left(H_{1}^{-1}\left(z_{2}(\xi)\right)\right)\right)} d \xi
$$

From (31), the above inequality can be written as

$$
\begin{aligned}
H_{2}\left(H_{1}^{-1}\left(z_{2}(t)\right)\right) \leq & H_{2}\left(H_{1}^{-1}\left(A_{2}(t)\right)\right)+\int_{t_{0}}^{t} g_{2}(T, \xi) d \xi \\
& +\sum_{j=2}^{3} \int_{t_{0}}^{t} \frac{g_{j+1}(T, \xi) y_{j+1}\left(\varphi^{-1}\left(H_{1}^{-1}\left(z_{2}(\xi)\right)\right)\right)}{y_{2}\left(\varphi^{-1}\left(H_{1}^{-1}\left(z_{2}(\xi)\right)\right)\right)} d \xi .
\end{aligned}
$$

Let

$$
\eta_{2}(t)=H_{2}\left(H_{1}^{-1}\left(z_{2}(t)\right)\right)
$$

and

$$
A_{3}(t)=H_{2}\left(H_{1}^{-1}\left(A_{2}(t)\right)\right)+\int_{t_{0}}^{t} g_{2}(T, \xi) d \xi
$$

thus $H_{1}^{-1}\left(z_{2}(t)\right)=H_{2}^{-1}\left(\eta_{2}(t)\right)$, and by (31) and (32), the inequality (31) can be written as

$$
\begin{aligned}
\eta_{2}(t) & \leq A_{3}(t)+\sum_{j=2}^{3} \int_{t_{0}}^{t} \frac{g_{j+1}(T, \xi) y_{j+1}\left(\varphi^{-1}\left(H_{1}^{-1}\left(z_{2}(\xi)\right)\right)\right)}{y_{2}\left(\varphi^{-1}\left(H_{1}^{-1}\left(z_{2}(\xi)\right)\right)\right)} d \xi \\
& =A_{3}(t)+\sum_{j=2}^{3} \int_{t_{0}}^{t} \frac{g_{j+1}(T, \xi) y_{j+1}\left(\varphi^{-1}\left(H_{2}^{-1}\left(\eta_{2}(\xi)\right)\right)\right)}{y_{2}\left(\varphi^{-1}\left(H_{2}^{-1}\left(\eta_{2}(\xi)\right)\right)\right)} d \xi .
\end{aligned}
$$

Again, we assume that

$$
z_{3}(t)=A_{3}(t)+\sum_{j=2}^{3} \int_{t_{0}}^{t} \frac{g_{j+1}(T, \xi) y_{j+1}\left(\varphi^{-1}\left(H_{2}^{-1}\left(\eta_{2}(\xi)\right)\right)\right)}{y_{2}\left(\varphi^{-1}\left(H_{2}^{-1}\left(\eta_{2}(\xi)\right)\right)\right)} d \xi
$$

we can see that $z_{3}(t)$ is a nondecreasing function on $\left[t_{0}, t\right]$, and $\eta_{2}(t) \leq z_{3}(t), z_{3}(t) \geq A_{3}(t)$, $z_{3}\left(t_{0}\right)=A_{3}\left(t_{0}\right)$. Differentiating $z_{3}(t)$ with respect to $t$, we can obtain

$$
\begin{aligned}
z_{3}{ }^{\prime}(t) & =A_{3}{ }^{\prime}(t)+\sum_{j=2}^{3} \frac{g_{j+1}(T, t) y_{j+1}\left(\varphi^{-1}\left(H_{2}^{-1}\left(\eta_{2}(t)\right)\right)\right)}{y_{2}\left(\varphi^{-1}\left(H_{2}^{-1}\left(\eta_{2}(t)\right)\right)\right)} \\
& \leq A_{3}{ }^{\prime}(t)+\sum_{j=2}^{3} \frac{g_{j+1}(T, t) y_{j+1}\left(\varphi^{-1}\left(H_{2}^{-1}\left(z_{3}(t)\right)\right)\right)}{y_{2}\left(\varphi^{-1}\left(H_{2}^{-1}\left(z_{3}(t)\right)\right)\right)},
\end{aligned}
$$


multiplying by $\frac{y_{2}\left(\varphi^{-1}\left(H_{2}^{-1}\left(z_{3}(t)\right)\right)\right)}{y_{3}\left(\varphi^{-1}\left(H_{2}^{-1}\left(z_{3}(t)\right)\right)\right)}$, then integrating both sides from $t_{0}$ to $t$, and using $\left(C_{2}\right)$, we can obtain

$$
\begin{aligned}
\int_{t_{0}}^{t} \frac{y_{2}\left(\varphi^{-1}\left(H_{2}^{-1}\left(z_{3}(\xi)\right)\right)\right)}{y_{3}\left(\varphi^{-1}\left(H_{2}^{-1}\left(z_{3}(\xi)\right)\right)\right)} z_{3}{ }^{\prime}(\xi) d \xi \leq & \int_{t_{0}}^{t} \frac{y_{2}\left(\varphi^{-1}\left(H_{2}^{-1}\left(z_{3}(\xi)\right)\right)\right)}{y_{3}\left(\varphi^{-1}\left(H_{2}^{-1}\left(z_{3}(\xi)\right)\right)\right)} A_{3}{ }^{\prime}(\xi) d \xi+\int_{t_{0}}^{t} g_{3}(T, \xi) d \xi \\
& +\int_{t_{0}}^{t} \frac{g_{4}(T, \xi) y_{4}\left(\varphi^{-1}\left(H_{2}^{-1}\left(z_{3}(\xi)\right)\right)\right)}{y_{3}\left(\varphi^{-1}\left(H_{2}^{-1}\left(z_{3}(\xi)\right)\right)\right)} d \xi
\end{aligned}
$$

then

$$
\begin{aligned}
Y_{3}\left(z_{3}(t)\right)-Y_{3}\left(z_{3}\left(t_{0}\right)\right) \leq & \int_{t_{0}}^{t} \frac{y_{2}\left(\varphi^{-1}\left(H_{2}^{-1}\left(A_{3}(\xi)\right)\right)\right)}{y_{3}\left(\varphi^{-1}\left(H_{2}^{-1}\left(A_{3}(\xi)\right)\right)\right)} A_{3}{ }^{\prime}(\xi) d \xi+\int_{t_{0}}^{t} g_{3}(T, \xi) d \xi \\
& +\int_{t_{0}}^{t} \frac{g_{4}(T, \xi) y_{4}\left(\varphi^{-1}\left(H_{2}^{-1}\left(z_{3}(\xi)\right)\right)\right)}{y_{3}\left(\varphi^{-1}\left(H_{2}^{-1}\left(z_{3}(\xi)\right)\right)\right)} d \xi \\
\leq & Y_{3}\left(A_{3}(t)\right)-Y_{3}\left(A_{3}\left(t_{0}\right)\right)+\int_{t_{0}}^{t} g_{3}(T, \xi) d \xi \\
& +\int_{t_{0}}^{t} \frac{g_{4}(T, \xi) y_{4}\left(\varphi^{-1}\left(H_{2}^{-1}\left(z_{3}(\xi)\right)\right)\right)}{y_{3}\left(\varphi^{-1}\left(H_{2}^{-1}\left(z_{3}(\xi)\right)\right)\right)} d \xi,
\end{aligned}
$$

by $z_{3}\left(t_{0}\right)=A_{3}\left(t_{0}\right)$, we can obtain

$$
Y_{3}\left(z_{3}(t)\right) \leq Y_{3}\left(A_{3}(t)\right)+\int_{t_{0}}^{t} g_{3}(T, \xi) d \xi+\int_{t_{0}}^{t} \frac{g_{4}(T, \xi) y_{4}\left(\varphi^{-1}\left(H_{2}^{-1}\left(z_{3}(\xi)\right)\right)\right)}{y_{3}\left(\varphi^{-1}\left(H_{2}^{-1}\left(z_{3}(\xi)\right)\right)\right)} d \xi .
$$

Using (31), we can obtain

$$
\begin{aligned}
H_{3}\left(H_{2}^{-1}\left(z_{3}(t)\right)\right) \leq & H_{3}\left(H_{2}^{-1}\left(A_{3}(t)\right)\right)+\int_{t_{0}}^{t} g_{3}(T, \xi) d \xi \\
& +\int_{t_{0}}^{t} \frac{g_{4}(T, \xi) y_{4}\left(\varphi^{-1}\left(H_{2}^{-1}\left(z_{3}(\xi)\right)\right)\right)}{y_{3}\left(\varphi^{-1}\left(H_{2}^{-1}\left(z_{3}(\xi)\right)\right)\right)} d \xi .
\end{aligned}
$$

Again, let

$$
\begin{aligned}
& \eta_{3}(t)=H_{3}\left(H_{2}^{-1}\left(z_{3}(t)\right)\right), \\
& A_{4}(t)=H_{3}\left(H_{2}^{-1}\left(A_{3}(t)\right)\right)+\int_{t_{0}}^{t} g_{3}(T, \xi) d \xi,
\end{aligned}
$$

thus $H_{2}^{-1}\left(z_{3}(t)\right)=H_{3}^{-1}\left(\eta_{3}(t)\right)$, using (35) and (36), the inequality (34) can be written as

$$
\begin{aligned}
\eta_{3}(t) & \leq A_{4}(t)+\int_{t_{0}}^{t} \frac{g_{4}(T, \xi) y_{4}\left(\varphi^{-1}\left(H_{2}^{-1}\left(z_{3}(\xi)\right)\right)\right)}{y_{3}\left(\varphi^{-1}\left(H_{2}^{-1}\left(z_{3}(\xi)\right)\right)\right)} d \xi \\
& =A_{4}(t)+\int_{t_{0}}^{t} \frac{g_{4}(T, \xi) y_{4}\left(\varphi^{-1}\left(H_{3}^{-1}\left(\eta_{3}(\xi)\right)\right)\right)}{\left.y_{3}\left(\varphi^{-1}\left(H_{3}^{-1}\left(\eta_{3}(\xi)\right)\right)\right)\right)} d \xi .
\end{aligned}
$$

We assume that

$$
z_{4}(t)=A_{4}(t)+\int_{t_{0}}^{t} \frac{g_{4}(T, \xi) y_{4}\left(\varphi^{-1}\left(H_{3}^{-1}\left(\eta_{3}(\xi)\right)\right)\right)}{\left.y_{3}\left(\varphi^{-1}\left(H_{3}^{-1}\left(\eta_{3}(\xi)\right)\right)\right)\right)} d \xi
$$


then we see that $z_{4}(t)$ is a nondecreasing function on $\left[t_{0}, t\right]$, and $\eta_{3}(t) \leq z_{4}(t), z_{4}(t) \geq A_{4}(t)$, $z_{4}\left(t_{0}\right)=A_{4}\left(t_{0}\right)$.

Differentiating (37) with respect to $t$, we can obtain

$$
z_{4}{ }^{\prime}(t)=A_{4}{ }^{\prime}(t)+\frac{g_{4}(T, t) y_{4}\left(\varphi^{-1}\left(H_{3}^{-1}\left(\eta_{3}(t)\right)\right)\right)}{\left.y_{3}\left(\varphi^{-1}\left(H_{3}^{-1}\left(\eta_{3}(t)\right)\right)\right)\right)} \leq A_{4}{ }^{\prime}(t)+\frac{g_{4}(T, t) y_{4}\left(\varphi^{-1}\left(H_{3}^{-1}\left(z_{4}(t)\right)\right)\right)}{\left.y_{3}\left(\varphi^{-1}\left(H_{3}^{-1}\left(z_{4}(t)\right)\right)\right)\right)} .
$$

Now, multiplying both sides of it by $\frac{y_{3}\left(\varphi^{-1}\left(H_{3}^{-1}\left(z_{4}(t)\right)\right)\right)}{y_{4}\left(\varphi^{-1}\left(H_{3}^{-1}\left(z_{4}(t)\right)\right)\right.}$, then integrating both sides of it from $t_{0}$ to $t$, and using $\left(C_{2}\right)$, we can obtain

$$
\int_{t_{0}}^{t} \frac{y_{3}\left(\varphi^{-1}\left(H_{3}^{-1}\left(z_{4}(\xi)\right)\right)\right)}{y_{4}\left(\varphi^{-1}\left(H_{3}^{-1}\left(z_{4}(\xi)\right)\right)\right)} z_{4}{ }^{\prime}(\xi) d \xi \leq \int_{t_{0}}^{t} \frac{y_{3}\left(\varphi^{-1}\left(H_{3}^{-1}\left(z_{4}(\xi)\right)\right)\right)}{y_{4}\left(\varphi^{-1}\left(H_{3}^{-1}\left(z_{4}(\xi)\right)\right)\right)} A_{4}{ }^{\prime}(\xi) d \xi+\int_{t_{0}}^{t} g_{4}(T, \xi) d \xi
$$

thus

$$
\begin{aligned}
Y_{4}\left(z_{4}(t)\right)-Y_{4}\left(z_{4}\left(t_{0}\right)\right) & \leq \int_{t_{0}}^{t} \frac{y_{3}\left(\varphi^{-1}\left(H_{3}^{-1}\left(A_{4}(\xi)\right)\right)\right)}{y_{4}\left(\varphi^{-1}\left(H_{3}^{-1}\left(A_{4}(\xi)\right)\right)\right)} A_{4}{ }^{\prime}(\xi) d \xi+\int_{t_{0}}^{t} g_{4}(T, \xi) d \xi \\
& \leq Y_{4}\left(A_{4}(t)\right)-Y_{4}\left(A_{4}\left(t_{0}\right)\right)+\int_{t_{0}}^{t} g_{4}(T, \xi) d \xi
\end{aligned}
$$

by $z_{4}\left(t_{0}\right)=A_{4}\left(t_{0}\right)$, we can obtain

$$
Y_{4}\left(z_{4}(t)\right) \leq Y_{4}\left(A_{4}(t)\right)+\int_{t_{0}}^{t} g_{4}(T, \xi) d \xi
$$

Using the definition of (31), we can get

$$
H_{4}\left(H_{3}^{-1}\left(z_{4}(t)\right)\right) \leq H_{4}\left(H_{3}^{-1}\left(A_{4}(t)\right)\right)+\int_{t_{0}}^{t} g_{4}(T, \xi) d \xi,
$$

thus

$$
z_{4}(t) \leq H_{3}\left[H_{4}^{-1}\left(H_{4}\left(H_{3}^{-1}\left(A_{4}(t)\right)\right)+\int_{t_{0}}^{t} g_{4}(T, \xi) d \xi\right)\right] .
$$

Using (27), (31), (33), (35) and (38), we can obtain

$$
\begin{aligned}
z_{1}(t) & =H_{1}^{-1}\left(\eta_{1}(t)\right) \leq H_{1}^{-1}\left(z_{2}(t)\right)=H_{2}^{-1}\left(\eta_{2}(t)\right) \leq H_{2}^{-1}\left(z_{3}(t)\right)=H_{3}^{-1}\left(\eta_{3}(t)\right) \\
& \leq H_{3}^{-1}\left(z_{4}(t)\right) \leq H_{4}^{-1}\left(H_{4}\left(H_{3}^{-1}\left(A_{4}(t)\right)\right)+\int_{t_{0}}^{t} g_{4}(T, \xi) d \xi\right)
\end{aligned}
$$

then we have

$$
\begin{aligned}
u(t) & \leq \varphi^{-1}\left(z_{1}(t)\right) \\
& \leq \varphi^{-1}\left[H_{4}^{-1}\left(H_{4}\left(H_{3}^{-1}\left(A_{4}(t)\right)\right)+\int_{t_{0}}^{t} g_{4}(T, \xi) d \xi\right)\right] \\
& \leq \varphi^{-1}\left(H_{4}^{-1}\left(A_{5}(t)\right)\right), \quad t \in\left[t_{0}, T\right],
\end{aligned}
$$


where $A_{5}(t)=H_{4}\left(H_{3}^{-1}\left(A_{4}(t)\right)\right)+\int_{t_{0}}^{t} g_{4}(T, \xi) d \xi$, because of $T$ being arbitrary, we can obtain

$$
\begin{aligned}
u(t) & \leq \varphi^{-1}\left(z_{1}(t)\right) \\
& \leq \varphi^{-1}\left[H_{4}^{-1}\left(H_{4}\left(H_{3}^{-1}\left(A_{4}(t)\right)\right)+\int_{t_{0}}^{t} g_{4}(t, \xi) d \xi\right)\right] \\
& \leq \varphi^{-1}\left(H_{4}^{-1}\left(A_{5}(t)\right)\right), \quad t \in\left[t_{0}, T\right] .
\end{aligned}
$$

The proof is complete.

Remark 3 If $h_{3} \equiv 0$, we can see that Theorem 3.3 reduces to Theorem 2.3 in [9]. If $\varphi(u(t))=x^{p}(t), a(t)=x_{0}, g_{1}(t, \xi)=f(s), h_{1}(u(t))=x^{p}(t), g_{2}(t, \xi)=h(s), h_{2}(u(t))=x^{q}(t)$, and $g(t, \xi)=g_{3}(t, \xi)=0$, Theorem 3.3 reduces to Theorem 3.1 in [8]. If $h_{1}(u(t))=\eta(u(s)) w(u(s))$, $h_{2}(u(t))=\eta(u(s))$, and $g(t, \xi)=g_{3}(t, \xi)=0$, Theorem 3.3 reduces to Theorem 1 in [12].

\section{Applications of the result}

In this section, we apply the results of the previous section to study the boundedness of solutions of differential equations and integral equations.

1. First, let us consider the Volterra type retarded integral equation

$$
\chi^{4}(t)=b(t)+\int_{t_{0}}^{\sigma(t)} g(\xi)\left[\chi^{2}(\xi)+\int_{t_{0}}^{\xi} w(\lambda) \chi^{2}(\lambda) d \lambda\right]^{\frac{1}{3}} d \xi
$$

which often occurs in physical and mechanical applications.

Example 4.1 We assume $\chi(t), b(t), g(t), w(t) \in C\left(R_{+}, R_{+}\right)$, and let $\sigma(t) \in C\left[t_{0}, \infty\right), \sigma^{\prime}(t) \geq 0$ and $\sigma(t) \leq t, \sigma\left(t_{0}\right)=t_{0}$. We can obtain the estimate for $\chi(t)$ as follows:

$$
|\chi(t)| \leq\left[|b(t)|+B(t) \exp \left(\int_{t_{0}}^{\sigma(t)} \frac{1}{6}|g(\xi)| d \xi+\int_{t_{0}}^{\sigma(t)} \frac{1}{6}|g(\xi)|\left(\int_{t_{0}}^{s}|w(\lambda)| d \lambda\right) d \xi\right)\right]^{\frac{1}{4}}
$$

where

$$
\begin{aligned}
B(t)= & \int_{t_{0}}^{\sigma(t)}|g(\xi)|\left(\frac{2}{3}+\frac{1}{6}|b(\xi)|+\frac{1}{6}\right) d \xi \\
& +\int_{t_{0}}^{\sigma(t)}|g(\xi)|\left[\int_{t_{0}}^{\xi}|w(\lambda)|\left(\frac{1}{6}|b(\lambda)|+\frac{1}{6}\right) d \lambda\right] d \xi .
\end{aligned}
$$

Proof By (39), we have

$$
|\chi(t)|^{4} \leq|b(t)|+\int_{t_{0}}^{\sigma(t)}|g(\xi)|\left[|\chi(\xi)|^{2}+\int_{t_{0}}^{\xi}|w(\lambda)||\chi(\lambda)|^{2} d \lambda\right]^{\frac{1}{3}} d \xi
$$

taking $|\chi(t)|=u(t)$, (40) can be written as

$$
u^{4}(t) \leq|b(t)|+\int_{t_{0}}^{\sigma(t)}|g(\xi)|\left[u^{2}(t)+\int_{t_{0}}^{\xi}|w(\lambda)| u^{2}(\lambda) d \lambda\right]^{\frac{1}{3}} d \xi
$$


Here, we can conclude that (41) satisfies the conditions of Theorem 3.1 with $q=4, \alpha=$ $\beta=2, p=\frac{1}{3}, a(t)=|b(t)|, h(t)=|w(t)|, f(t)=|g(t)|$, using Theorem 3.1, our conclusion obviously holds.

2. Next, we consider the following integral equation:

$$
\chi(t)=\chi_{0}+\int_{t_{0}}^{\sigma(t)} f(\xi) \chi^{\frac{1}{5}}(\xi)\left[\chi^{3}(\xi)+\int_{t_{0}}^{\xi} w(\lambda) \chi^{3}(\lambda) d \lambda\right]^{4} d \xi
$$

Example 4.2 We assume $\chi(t), f(t), w(t) \in C\left(R_{+}, R_{+}\right), \chi^{\prime}(t) \geq 0, \sigma(t) \in\left[t_{0}, \infty\right), \sigma^{\prime}(t) \geq 0$, $\sigma(t) \leq t, \sigma\left(t_{0}\right)=t_{0}$, then we can get

$$
\begin{aligned}
|\chi(t)| \leq & \exp \left(\frac{1}{5} \int_{t_{0}}^{\sigma(t)}|f(\xi)| \beta(\xi) d \xi\right) \\
& \times\left[\chi_{0}+\int_{t_{0}}^{\sigma(t)}\left(\frac{4}{5}\right)|f(\xi)| \beta(\xi) \exp \left(-\frac{1}{5} \int_{t_{0}}^{\xi}|f(\lambda)| \beta(\lambda) d \lambda\right) d \xi\right],
\end{aligned}
$$

where

$$
\begin{aligned}
& \beta(t)=\left[\frac{\Omega(t)}{1-\left(\frac{64}{5}\right) \int_{t_{0}}^{t}|f(\xi)| \Omega(\xi) d \xi}\right]^{\frac{15}{14}}, \\
& \Omega(t)=\chi_{0}^{\frac{56}{5}} \exp \left(\frac{56}{15} \int_{t_{0}}^{t}|w(\xi)| d \xi\right) .
\end{aligned}
$$

Proof Using (42), we have

$$
|\chi(t)| \leq \chi_{0}+\int_{t_{0}}^{\sigma(t)}|f(\xi)||\chi(\xi)|^{\frac{1}{5}}\left[|\chi(\xi)|^{3}+\int_{t_{0}}^{\xi}|w(\lambda)||\chi(\lambda)|^{3} d \lambda\right]^{4} d \xi
$$

let $|\chi(t)|=u(t)$, the above inequality is written as

$$
u(t) \leq \chi_{0}+\int_{t_{0}}^{\sigma(t)}|f(\xi)| u^{\frac{1}{5}}(\xi)\left[u^{3}(\xi)+\int_{t_{0}}^{\xi}|w(\lambda)| u^{3}(\xi) d \lambda\right]^{4} d \xi
$$

Here, we can conclude that (44) satisfies the conditions of Theorem 3.2 with $m=m=3$, $p=4, r=\frac{1}{5}, u_{0}=\chi_{0}, h(t)=|w(t)|, g(t)=|f(t)|$, using Theorem 3.2, our conclusion obviously holds.

3. We consider the following differential system:

$$
\left\{\begin{array}{l}
s^{\prime}(t)=G(t, s), \quad t \in[0, \infty) \\
s(0)=a_{0}
\end{array}\right.
$$

where $G(t, s)$ is a continuous function on $[0, \infty) \times(-\infty,-e \sqrt{e}] \cup[e \sqrt{e},+\infty), a_{0}>0$. We assume that $G(t, s)$ satisfies the following inequality:

$$
|G(t, s)| \leq t^{2} \sqrt[5]{|s|}+\frac{|s|}{3}-\frac{|s| \ln |s|}{2}+\frac{e^{|s|}}{4}
$$


Example 4.3 Let $G(t, s)$ satisfy the condition of inequality (46), all solutions of differential system (45) satisfy the following estimates:

$$
|s(t)| \leq-\ln \left(\exp \left(-\left(\left(a_{0}^{\frac{4}{5}}+\frac{4 t^{3}}{15}\right)^{\frac{5}{4}} e^{\frac{t}{3}}\right)^{e^{-\frac{t}{2}}}\right)+\frac{t}{4}\right), \quad \forall t \in[0, \infty)
$$

Proof Integrating the differential system (45) from 0 to $t$, we can get

$$
s(t)=a_{0}+\int_{0}^{t} G(\xi, s(\xi)) d \xi
$$

by (46), we can obtain

$$
|s(t)| \leq a_{0}+\int_{0}^{t} \xi^{2} \sqrt[5]{|s(\xi)|} d \xi+\int_{0}^{t} \frac{|s(\xi)|}{3} d \xi-\int_{0}^{t} \frac{|s(\xi)| \ln |s(\xi)|}{2} d \xi+\int_{0}^{t} \frac{e^{|s|}}{4} d \xi
$$

taking $|s(t)|=u(t)$, the above inequality can be written as

$$
u(t) \leq a_{0}+\int_{0}^{t} \xi^{2} \sqrt[5]{u(\xi)} d \xi+\int_{0}^{t} \frac{u(\xi)}{3} d \xi-\int_{0}^{t} \frac{u(\xi) \ln (u(\xi))}{2} d \xi+\int_{0}^{t} \frac{e^{|s|}}{4} d \xi
$$

we can see that Eq. (49) satisfies (24) : $a(t)=a_{0}, g_{1}(t, \xi)=t^{2}, g_{2}(t, \xi)=\frac{1}{3}, g_{3}(t, \xi)=-\frac{1}{2}$, $g_{4}(t, \xi)=\frac{1}{4}, h_{1}(u)=\sqrt[5]{|u|}, h_{2}(u)=|u|, h_{3}(u)=|u| \ln |u|, h_{4}(u)=e^{|s|}, \frac{h_{2}(t)}{h_{1}(t)}=\frac{|u|}{\sqrt[5]{|u|}}=|u|^{\frac{4}{5}}, \frac{h_{3}(t)}{h_{2}(t)}=$ $\ln |u|, \frac{h_{4}(t)}{h_{3}(t)}=\frac{e^{|s|}}{|u| \ln |u|}$, then we can see that $\frac{h_{j+1}(t)}{h_{j}(t)},(j=1,2,3)$ is a nondecreasing function for $u>0$. then we can obtain

$$
\begin{aligned}
& H_{1}(u)=\int_{u_{0}}^{u} \frac{d \xi}{\sqrt[5]{\xi}}=\frac{5}{4}\left(u^{\frac{4}{5}}-u_{0}^{\frac{4}{5}}\right), \quad H_{1}^{-1}(u)=\left(\frac{4}{5} u+u_{0}^{\frac{4}{5}}\right)^{\frac{5}{4}}, \\
& H_{2}(u)=\int_{u_{1}}^{u} \frac{d \xi}{\xi}=\ln \frac{u}{u_{1}}, \quad H_{2}^{-1}(u)=u_{1} e^{u}, \\
& H_{3}(u)=\int_{e}^{u} \frac{d \xi}{\xi \ln \xi}=\ln (\ln (u)), \quad H_{3}^{-1}(u)=e^{e^{u}}, \\
& H_{4}(u)=\int_{1}^{u} \frac{d \xi}{e^{\xi}}=-\left(e^{-u}-e^{-1}\right), \quad H_{4}^{-1}(u)=-\ln \left(e^{-1}-u\right) .
\end{aligned}
$$

Using Eq. (23) of Theorem 3.3, we have

$$
\begin{aligned}
A_{1}(t) & =a_{0}, \\
A_{2}(t) & =H_{1}\left(A_{1}(t)\right)+\int_{0}^{t} \xi^{2} d \xi \\
& =\frac{5}{4}\left(a_{0}^{\frac{4}{5}}-u_{0}^{\frac{4}{5}}\right)+\frac{t^{3}}{3}, \\
A_{3}(t) & =H_{2}\left(H_{1}^{-1}\left(A_{2}(t)\right)\right)+\int_{0}^{t} \frac{1}{3} d \xi \\
& =H_{2}\left[\left(a_{0}^{\frac{4}{5}}+\frac{4 t^{3}}{15}\right)^{\frac{5}{4}}\right]+\frac{t}{3}
\end{aligned}
$$




$$
\begin{aligned}
& =\ln \frac{\left(a_{0}^{\frac{4}{5}}+\frac{4 t^{3}}{15}\right)^{\frac{5}{4}}}{u_{1}}+\frac{t}{3}, \\
A_{4}(t) & =H_{3}^{-1}\left(H_{2}\left(A_{3}(t)\right)\right)-\int_{0}^{t} \frac{1}{2} d \xi \\
& =H_{3}\left[u_{1} \exp \left(\ln \frac{\left(a_{0}^{\frac{4}{5}}+\frac{4 t^{3}}{15}\right)^{\frac{5}{4}}}{u_{1}}+\frac{t}{3}\right)\right]-\frac{t}{2} \\
& =H_{3}\left[\left(a_{0}^{\frac{4}{5}}+\frac{4 t^{3}}{15}\right)^{\frac{5}{4}} e^{\frac{t}{3}}\right]-\frac{t}{2} \\
& =\ln \left[\ln \left(\left(a_{0}^{\frac{4}{5}}+\frac{4 t^{3}}{15}\right)^{\frac{5}{4}} e^{\frac{t}{3}}\right)\right]-\frac{t}{2}, \\
A_{5}= & H_{4}\left[H_{3}^{-1}\left(A_{4}(t)\right)\right]+\int_{0}^{t} \frac{1}{4} d \xi \\
= & H_{4}\left[\left(\left(a_{0}^{\frac{4}{5}}+\frac{4 t^{3}}{15}\right)^{\frac{5}{4}} e^{\frac{t}{3}}\right)^{e^{-\frac{t}{2}}}\right]+\frac{t}{4} \\
= & e^{-1}-\exp \left(-\left(\left(a_{0}^{\frac{4}{5}}+\frac{4 t^{3}}{15}\right)^{\frac{5}{4}} e^{\frac{t}{3}}\right)^{e^{-\frac{t}{2}}}\right)+\frac{t}{4},
\end{aligned}
$$

then

$$
\begin{aligned}
u(t) & \leq H_{4}^{-1}\left(A_{5}(t)\right) \\
& =-\ln \left(\exp \left(-\left(\left(a_{0}^{\frac{4}{5}}+\frac{4 t^{3}}{15}\right)^{\frac{5}{4}} e^{\frac{t}{3}}\right)^{e^{-\frac{t}{2}}}\right)+\frac{t}{4}\right),
\end{aligned}
$$

which means that $u(t)$ is bounded, for $t \in[0, \infty)$. The proof is completed.

\section{Conclusion}

In this paper, we first give a new lemma about the nonlinear Gronwall-Bellman delay integral inequality, then we establish some new delay Gronwall-Bellman integral inequalities with power. And the inequalities obtained in this paper are further generalizations of some results obtained by Li et al. [9]. The results of this paper contribute to the study of the qualitative properties of solutions of differential and integral equations. By the method of Theorem 3.3 in this paper, we can further generalize Eq. (3) to

$$
\begin{aligned}
\varphi(u(t)) \leq & a(t)+\sum_{j=1}^{n} \int_{t_{0}}^{t} g_{j}(t, \xi) h_{j}(u(\xi)) d \xi \\
& +\int_{t_{0}}^{t} g(t, \xi)\left(\int_{t_{0}}^{\xi} f(\xi, \theta) h_{n+1}(u(\theta)) d \theta\right) d \xi,
\end{aligned}
$$

then we can get similar results for the estimations on $u(t)$.

\section{Acknowledgements}

The authors would like to express their sincere thanks to the editor and anonymous reviewers for their helpful comments and suggestions. 


\section{Funding}

This research is supported by National Science Foundation of China $(11671227,11971015)$ and the Natural Science Foundation of Shandong Province (ZR2019MA034).

\section{Availability of data and materials}

We declare that the data and material in the paper can be used publicly.

\section{Competing interests}

The authors declare that they have no competing interests.

\section{Authors' contributions}

BF carried out the main results and completed the corresponding proof. YL participated in the proof of Theorem 3.2, RX participated in the proof and help to complete Sects. 4 and 5. All authors read and approved the final manuscript.

\section{Author details}

'School of Mathematical Science, Qufu Normal University, Qufu, China. ${ }^{2}$ School of Statistics, Qufu Normal University, Qufu, China.

\section{Publisher's Note}

Springer Nature remains neutral with regard to jurisdictional claims in published maps and institutional affiliations.

Received: 5 September 2020 Accepted: 10 February 2021 Published online: 07 May 2021

\section{References}

1. Gronwall, T.H.: Note on the derivatives with respect to a parameter of the solutions of a system of differential equations. Ann. Math. 20, 292-296 (1919)

2. Bellman, R.: The stability of solutions of linear differential equations. Duke Math. J. 10, 643-647 (1943)

3. Pachpatte, B.G.: On some generalizations of Bellman's lemma. J. Math. Anal. Appl. 51(1), 141-150 (1975)

4. Lakshmikantham, V., Leela, S.: Differential and Integral Inequalities, Theory and Applications. Academic Press, New York (1969)

5. El-Owaidy, H., Ragab, A., Abdeldaim, A.: On some new integral inequalities of Gronwall-Bellman type. Appl. Math. Comput. 106, 289-303 (1999)

6. Lipovan, O.: A retarded Gronwall-like inequality and its applications. J. Math. Anal. Appl. 252, 389-401 (2000)

7. Agarwal, R.P., Deng, S.F., Zhang, W.N.: Generalization of a retarded Gronwall-like inequality and its applications. Appl. Math. Comput. 165, 599-612 (2005)

8. Abdeldaim, A., Yakout, M.: On some new integral inequalities of Gronwall-Bellman-Pachpatte type. Appl. Math Comput. 217, 7887-7899 (2011)

9. Li, Z.Z., Wang, W.S.: Some nonlinear Gronwall-Bellman type retarded integral inequalities with power and their applications. Appl. Math. Comput. 347, 839-852 (2019)

10. El-Owaidy, H., Ragab, A., Abuelela, W., El-Deeb, A.A.: On some new nonlinear integral inequalities of Gronwall-Bellman type. Kyungpook Math. J. 54, 555-575 (2014)

11. Jiang, F.C., Meng, F.W.: Explicit bounds on some new nonlinear integral inequalities with delay. J. Comput. Appl. Math. 205, 479-486 (2007)

12. Ferreira, R.A.C., Torres, D.F.M.: Generalized retarded integral inequalities. Appl. Math. Lett. 22, 876-881 (2009)

13. Li, W.N., Han, M.A., Meng, F.W.: Some new delay integral inequalities and their applications. J. Comput. Appl. Math. 180, 191-200 (2005)

14. Pachpatte, B.G.: Inequalities for Differential and Integral Equations. Academic Press, London (1998)

15. Agarwal, R.P., Kim, Y.H., Sen, S.K.: New retarded integral inequalities with applications. J. Inequal. Appl. 2008, 908784 (2008)

16. Abdeldaim, A., El-Deeb, A.A.: On generalized of certain retarded nonlinear integral inequalities and its applications in retarded integro-differential equations. Appl. Math. Comput. 256, 375-380 (2015)

17. Bainov, D.D., Simeonov, P.: Integral Inequalities and Applications. Kluwer Academic, Dordrecht (1992)

18. Kuczma, M.: An Introduction to the Theory of Functional Equations and Inequalities: Cauchys Equation and Jensens Inequality. University of Katowice, Katowice (1985)

19. Li, W.N., Han, M.A., Meng, F.W.: Some new delay integral inequalities and their applications. J. Comput. Appl. Math. $180,191-200(2005)$

20. Li, Z.Z., Wang, W.S.: Explicit bounds of unknown function of some new weakly singular retarded integral inequalities for discontinuous functions and their applications. J. Inequal. Appl. 2017(287), 20 (2017)

21. Pachpatte, B.G.: Explicit bounds on certain integral inequalities. J. Math. Anal. Appl. 267, 48-61 (2002)

22. Feng, Q.H., Meng, F.W.: Some new Gronwall-type inequalities arising in the research of fractional differential equations. J. Inequal. Appl. 2013,429 (2013)

23. Shao, J., Meng, F.W.: Gronwall-Bellman type inequalities and their applications to fractional differential equations Abstr. Appl. Anal. 2013, Article ID 217641 (2013)

24. Medved, M.: Integral inequalities and global solutions of semilinear evolution equations. J. Math. Anal. Appl. 267, 643-650 (2002)

25. Xu, R., Meng, F.W.: Some new weakly singular integral inequalities and their applications to fractional differential equations. J. Inequal. Appl. 2016, 78 (2016)

26. Zhou, J., Shen, J., Zhang, W.N.: A powered Gronwall-type inequality and applications to stochastic differential equations. Discrete Contin. Dyn. Syst. 36(12), 7207-7234 (2016) 